\title{
Solar Energy Education at Grand Valley State University
}

\section{Dr. Lihong (Heidi) Jiao, Grand Valley State University}

Dr. Jiao is a Professor in the Padnos College of Engineering and Computing at Grand Valley State University. Her areas of interest include semiconductor device fabrication and characterization, nanomaterials, nano-devices, fiber optics, and nanotechnology education. Her research activities involve inorganic/organic solar cells, organic light emitting diodes, and MEMs/NEMs for sensor applications. 


\title{
Solar Energy Education at Grand Valley State University
}

\begin{abstract}
This paper presents the development of solar energy curriculum in the School of Engineering at Grand Valley State University. The aim of this curriculum is to provide students with fundamental theory and practical hands-on experiences. Three learning modules were developed that can be incorporated in the courses in the electrical engineering program. Two courses, one at sophomore level and one at senior level, have been identified for this purpose. A full course on photovoltaic systems was developed for the electrical engineering graduate program. The solar energy curriculum is supplemented by the SolaRescue program and Alternative Energy Club.
\end{abstract}

\section{Introduction}

Fossil fuels such as coal, oil, and natural gas have been the main energy sources since the beginning of human civilization. Even though fossil fuels are easier to transport and can generate energy efficiently, they are non-renewable and environmentally unfriendly. As a result, more and more efforts have been made to explore alternative energy sources such as solar and wind. Solar energy provides the potential for a clean, reliable, and more sustainable energy future. Historically, the photovoltaic (PV) industry has suffered from high initial installation costs. However, with declining PV module prices, the utility-scale PV system prices have been greatly decreasing; in some regions the price dropped to below $\$ 1.00$ per watt in the first half of $2017^{1}$. The decreased pricing of the PV systems enabled an annual global PV installation increase of 74 GWatt, reaching 299 GWatt at the end of 2016. The United States installed the second most PV capacity in 2016 and is one of the top markets in cumulative capacity. It is estimated that 380 GWatt of PV will be installed globally from 2017 to 2020, more than doubling currently installed capacity $^{2}$. The increased PV installations have led to significant job creation. The global PV sector employed 3.1 million people in 2016, a $12 \%$ increase from $2015^{3}$. It is estimated that the PV sector employment will increase at a rate of 20 times faster than that of the overall economy. It is essential that the workforce be educated to meet the needs of the PV industry. This paper describes the newly developed solar energy curriculum in the School of Engineering at Grand Valley State University (GVSU). The aim of this curriculum is to provide students with fundamental theory and practical hands-on experiences that will enable them to succeed in the field of solar PV.

Three learning modules have been developed that can be incorporated in courses in the electrical engineering program. Two courses have been identified for this purpose. One is the sophomore level course titled "Electronic Materials and Devices" and the other is the senior level course titled "Embedded Systems Interface". A course titled "Photovoltaic Systems" is developed for the graduate electrical engineering program, in which each component of the PV system is studied, and a significant project is assigned to design and build different PV systems. The solar energy curriculum is supplemented by the SolaRescue program and Alternative Energy Club. 
The details of the solar energy curriculum are presented in this paper along with both formative and summative assessments of the outcomes.

\section{Learning Modules on Solar Energy}

Three learning modules on solar energy have been developed which can be incorporated into courses in the electrical engineering program. The modules are solar radiation, solar cell, and solar energy harvesting.

\subsection{Solar Radiation Module}

Solar energy is clean, abundant, and renewable. To use the sun as an energy source, it is essential to know solar radiation, the sun's position for a given day and a location, and how to orient the solar panel to track the sun. Topics in this module include:
a. Solar irradiance, spectral irradiance, solar irradiation
b. Effects of atmosphere on solar radiation
c. Relationship between solar time and local time
d. Zenith angle, azimuth angle, and sun's position
e. How to orient solar panels to receive maximum solar energy available
f. A trade off between performance and cost

The module includes an activity where students are asked to write a program to track sun's position for a given date, time, and location. An example is shown in figure 1 where the sun's elevation angle throughout a day is shown for Grand Rapids, Mi on May $29^{\text {th }}$.

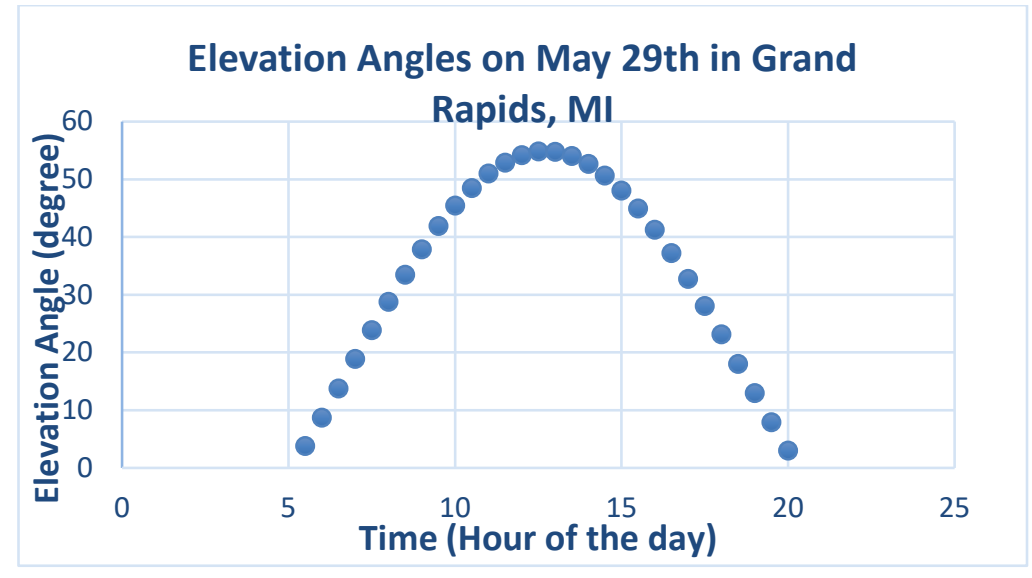

Figure 1 Elevation angle at different hours on May 29th in Grand Rapids, MI

\subsection{Solar Cell Module}

Solar energy can be converted either to heat energy (photo-thermal) or to electrical energy (photovoltaic). Since electricity is used widely and can be transported easily, photovoltaics is a focus here. The essential part of a photovoltaic system is the solar panel which is built from 
individual solar cells. This learning module studies the characteristics of solar cells. Topics include:
a. Solar cell structures
b. Solar cell current - voltage characteristics
c. Open circuit voltage, short circuit current, maximum power point, voltage and current at maximum power point
d. Fill factor and energy conversion efficiency
e. Measurement of solar cell parameters
f. Temperature and parasitic resistance effects on solar cell performance

Two activities are included in this module. One is to characterize silicon-based solar cells and the other is to make and characterize a nanocrystalline dye-sensitized solar cell (DSSC).

\subsubsection{Activity 1 - Silicon-based solar cell characterization}

The aim of this activity is to help students get familiar with different properties of silicon-based solar cells through measurements of solar cell parameters. The main apparatus needed are solar simulator, calibration cell, load resistor, and digital multimeter (DMM).

The solar simulator includes a light source, heated sample stage, and sample probes, all installed inside a dark chamber, of which the selection of the light source is of a great concern. It is vital to choose the light source that closely resembles the solar spectrum. Both Xenon arc lamp and halogen lamp are commonly used. Halogen light source (ELH lamp) is selected here due to its greater temporal stability and cost advantage over Xenon arc lamp. To better match the AM1.5 solar spectrum, infrared filters are inserted in the light path to filter out the excess infrared component. The AM1.5 light intensity is obtained through calibration using the reference cell. Neutral filters can be inserted in the light path to reduce the light intensity. The simulator chamber can be seen in figure 2 .

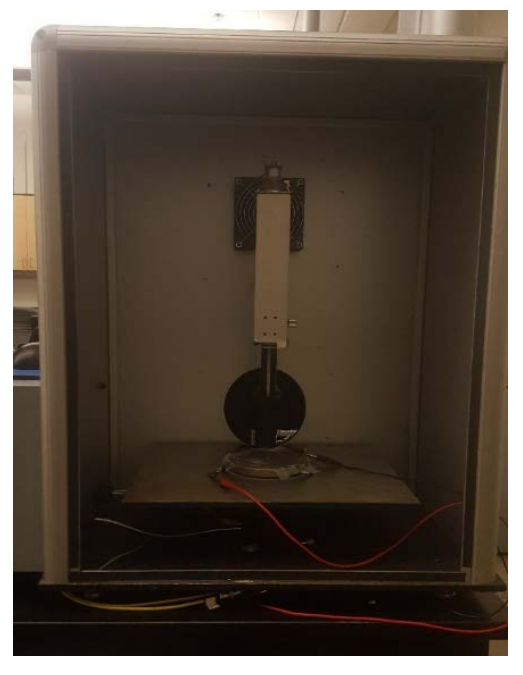

Figure 2 ELH solar simulator

The solar cell under measurement is placed on the sample stage and is connected to the external circuit shown in figure 3 for measurements of solar cell parameters. By adjusting the load resistance $\mathrm{R}$, the current and voltage of the solar cell can be measured from which the voltage and current at maximum power point, fill factor, and the energy conversion efficiency can be determined. By changing the light intensity and the sample temperature, their effects on the conversion efficiency are studied. 


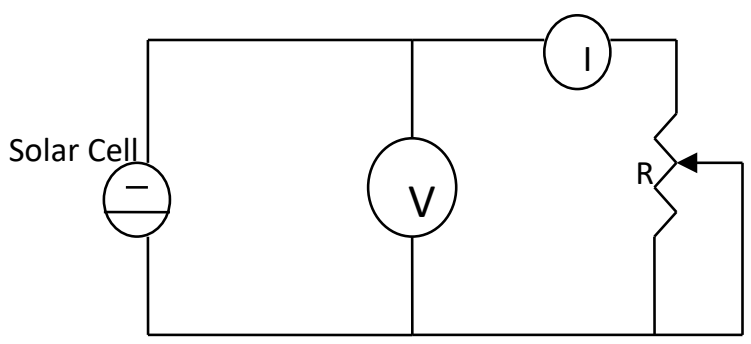

Figure 3 Circuit for solar cell measurements

\subsubsection{Activity 2 - Nanocrystalline dye-sensitized solar cells (DSSCs) ${ }^{4}$}

The aim of this activity is to synthesize a nanocrystalline dye-sensitized DSSC and measure the energy conversion efficiency of the synthesized solar cell.

Nanocrystalline DSSCs provide a good solution for low cost solar cell manufacturing. They are processed at room temperature and do not utilize high vacuum chambers. They operate on the principle of photosynthesis. A typical DSSC has three main layers as shown in figure 4: the photoanode, the electrolyte, and the photocathode. The photoanode is a working or active electrode which is composed of a layer of transparent conductive oxide such as $\mathrm{SnO}_{2}$, a layer of conductive nanocrystalline material such as $\mathrm{TiO}_{2}$, and the dye that can absorb light energy and create free moving electrons. The electrolyte is used to replenish the lost electrons in the dye. The photocathode serves as a counter electrode to supply electrons to the electrolyte.

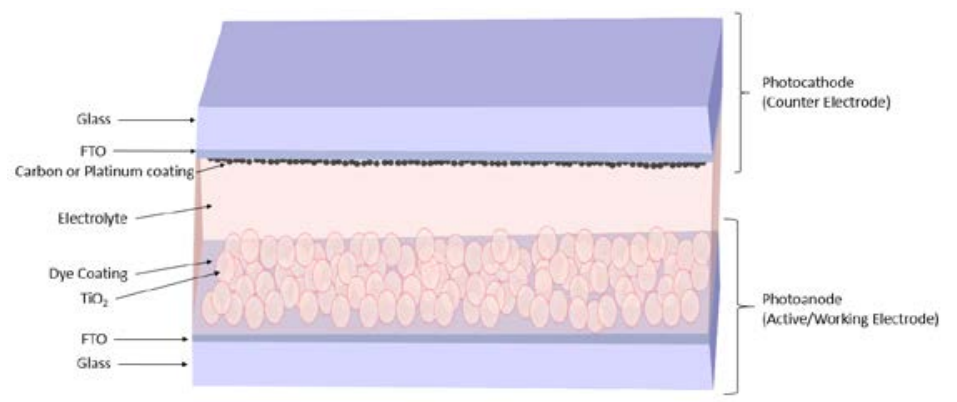

Figure 4 The structure of a typical DSSC

Materials used in this activity include: 2 pieces of FTO transparent glasses, $\mathrm{TiO}_{2}$ nanoparticles, Iodide electrolyte solution, raspberries, glass rod, scotch tape, Isopropanol alcohol. Petri dish, wash bottle, cotton swab, candle, binder clips, copper tape, hotplate, safety goggles, DMM potentiometer, and light source.

The photo of a synthesized DSSC is shown in figure 5 . The main synthesis processes are listed below. 
1) Deposition of a nano-porous $\mathrm{TiO}_{2}$ layer on FTO glass by doctor-blade method

2) Staining the $\mathrm{TiO}_{2}$ layer with the dye (raspberry)

3) Carbon-coating the counter electrode

4) Assembling the DSSC

5) Applying the electrolyte

6) Measuring the current and voltage at different load resistance

7) Determining the energy conversion efficiency

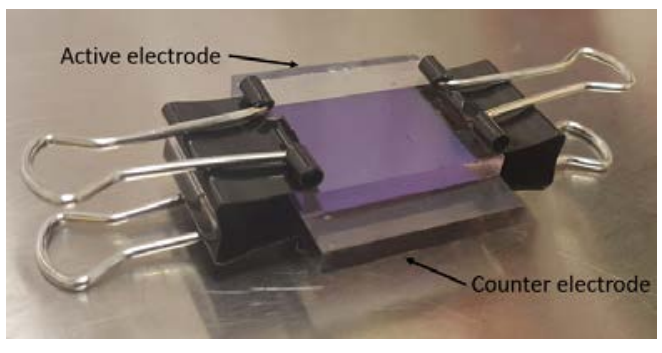

Figure 5 The synthesized nanocrystalline dyesensitized solar cell

\subsection{Solar Energy Harvesting Module}

Electrical energy generated by the solar cell needs to be collected efficiently and converted to a proper form using a specific conditioning circuit. The block diagram of a typical solar energy harvesting system, used to power a wireless network sensor node, is shown in figure 6 . The conditioning circuit regulates the power collected from the solar cell/panel, provides power to the network node, and charges an energy storage unit such as a rechargeable battery. The energy storage unit is required to power the system when the solar energy is unavailable. This learning module studies the functions of the solar energy harvesting system. Topics include:

a. Requirements of a solar energy harvesting system

b. DC/DC conversion circuit

c. Maximum power point tracking algorisms

d. Solar energy harvesting IC selection

e. Design and build a solar energy conditioning circuit that is used to charge a rechargeable Li-ion battery and to power an embedded system.

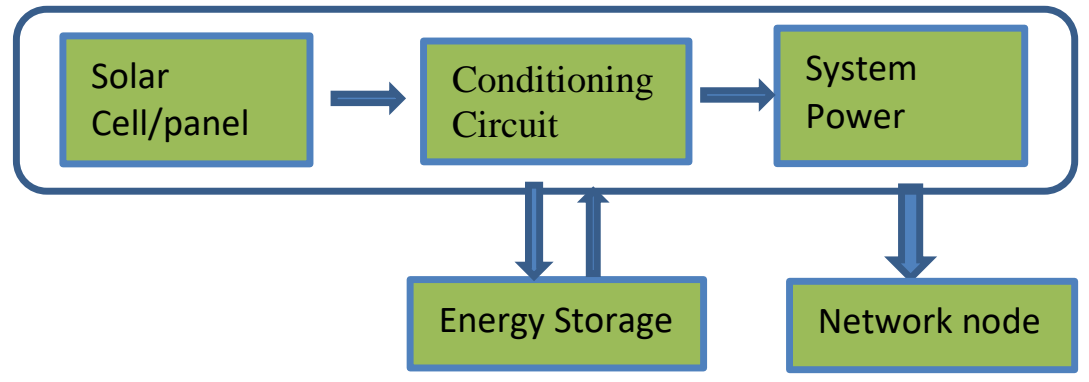

Figure 6 The block diagram of a typical solar energy harvester

The IC chip LTC3106 from Linear Technologies was selected to be the core of the conditioning circuit ${ }^{5}$. Students are required to study the datasheet of LTC3106 and design the conditioning circuit for a 4.8V/50mA solar module from PowerFilm. The main design requirements are listed below. 
1) The system voltage provided by the conditioning circuit can be selected from $2.2 \mathrm{~V}, 3.3 \mathrm{~V}$, and $5 \mathrm{~V}$.

2) A coin rechargeable Li ion battery should be incorporated and charging/discharging profiles should be properly managed.

3) Maximum power point tracking should be implemented.

4) The following terms should be monitored and displayed:

a. The voltage and current of the solar module

b. The voltage and current of the output to the system load

c. The temperature of the battery.

The schematic diagram of a sample conditioning circuit is shown in figure 7 . The circuit takes input from the solar cell/module, converts the input voltage either up or down based on the system voltage requirements, and charges the Li-ion battery if there is excess power. The circuit implements the maximum power point tracking to maximize the collected solar power. The Li-ion battery powers the system when the input solar power is not available.

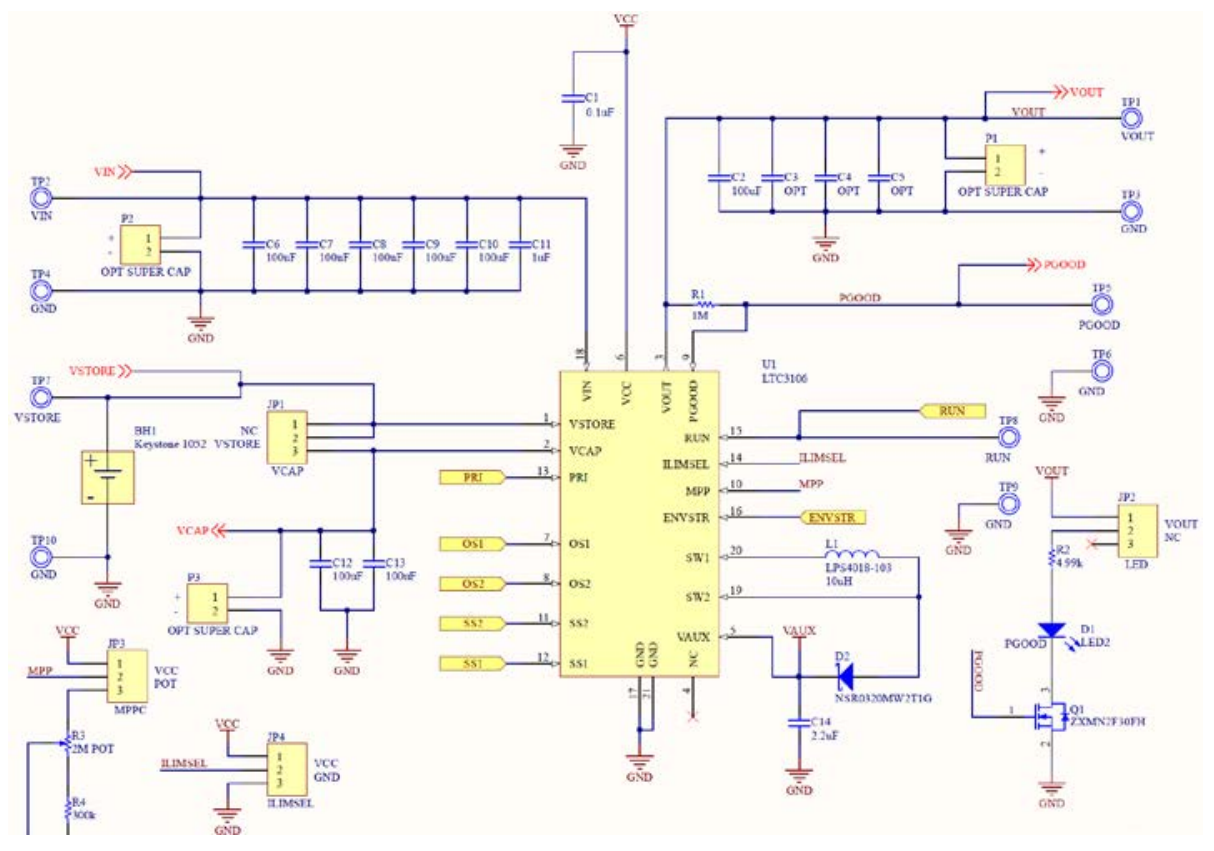

Figure 7 The schematic of LTC3106 based solar conditioning circuit

\section{Identifying Courses for the Learning Modules}

Two courses in our electrical engineering undergraduate curriculum were selected to incorporate these learning modules. One is EGR257 Electronic Materials and Devices, a sophomore level course; the other is EGR436 Embedded Systems Interface, a senior level elective course.

EGR257 teaches the properties of electronic materials and devices. Students learn the behavior of electrons and holes in semiconductors, how light interacts with semiconductors, p-n junction, diodes, BJTs, and MOSFETs. P-n junction is the foundation of all semiconductor devices. 
Silicon-based solar cell is a good example to show students the real world application of p-n junction diodes.

After learning the current - voltage (I-V) characteristics of a p-n junction at dark and under light illumination, photodiodes, LEDs, and solar cells are introduced. One class period is dedicated to the solar cell characterization module to discuss solar cell structures, parasitic series and parallel resistance, maximum power point, energy conversion efficiency, and solar cell measurements. One 1.5-hour lab period is assigned to activity 1 where students gain practical experiences on setting up solar simulators, measuring I-V characteristics of a solar cell, finding the maximum power point, and determining the energy conversion efficiency. Students also get a practical view of the effects of light intensity and sample temperature on the behavior of solar cells. One 3-hour lab period is assigned to activity 2 where different solar cell technologies are introduced. Students get an opportunity to make and characterize a solar cell and compare its characteristics with those of a silicon solar cell. Students get excited to see a running motor driven by their solar cell.

EGR436 teaches embedded systems interface. Embedded systems are found in virtually every modern electronic device. The core of the embedded system is a microcontroller. Embedded systems interface connects the microcontroller to sensors and other microprocessors through either wired or wireless communication networks directly or through Internet. In many remote wireless sensor nodes, batteries are used to supply electrical power. These batteries have limited lifetime and need to be replaced every few years. It is desired to have an autonomous wireless sensor node where electrical power is generated locally by an energy harvesting system.

The solar energy harvesting module is introduced in the second course project, wireless weather station. The objective of this project is to measure temperature, light intensity, humidity, barometric pressure and send them to the user through a wireless communication link. To make it an autonomous system, a solar energy harvesting system based on LTC3106 is included to supply electrical power to the wireless weather station.

\section{Photovoltaic Systems Course}

"Photovoltaic Systems" course has been developed for the electrical engineering graduate program. The goal of the course is to introduce students to the field of photovoltaics. The course covers the architectures of different PV systems and system components such as solar panels, energy storage units, charge controllers, DC/DC converters, inverters, etc. It provides technical, practical, and economic considerations in designing PV systems.

The objectives of the course are listed below.

1) Students will be able to determine the power produced by the PV panel as a function of module tilt and azimuth angle. 
2) Students will be able to describe the basic components and main performance parameters of a PV system.

3) Students will be able to design, troubleshoot, and test PV systems.

4) Students will be able to effectively communicate technical concepts.

The outline of the course are:

1) Semiconductor physics review

2) Photo emission and absorption

3) Solar cell characteristics and technologies

4) Solar radiation

5) PV system basics and design

6) PV system components

7) Applications and economics of PV systems

To provide students with hands-on and real-world experiences, a major course project is assigned $^{6}$. The course project is solicited from both for-profit and non-profit organizations. The objectives of the project are to design and build a PV system, to introduce students to the process of product design and realization, and more specifically, (1) to generate system requirements, specification documents, design documents, and test plans; (2) to properly size system components for a specific location; and (3) to test and troubleshoot a PV system. One example project, shown in figure 8 , is to develop a photovoltaic emergency power system (PVEPS) for the Embangweni hospital in Malawi. The PVEPS has been installed in the hospital and provides lighting, suction function, and

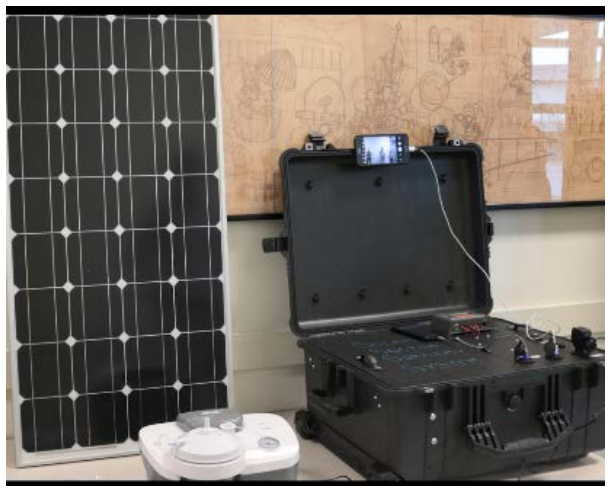

Figure 8 The photo of the PVEPS power to cell phones.

\section{Other Components}

The solar energy curriculum is supplemented by the SolaRescue program and Alternative Energy Club. The SolaRescue program was established in 2016. It is comprised of students, alumni and faculty, who share a passion for providing access to free resources to better lives of others. Its missions are to save lives with solar energy, to provide reliable energy for those left in the dark, and to use technology to help people in need. We strive to develop solutions for areas with limited grid power. The goal of the Alternative Energy Club is to incorporate alternative sources of energy into practical engineering applications and projects. One of the current projects is to build a solar powered car to race in the American Solar Challenge. 


\section{Assessment}

Both formative and summative assessments were conducted. At the beginning of each lecture or lab activity, questions related to the previous lesson were asked and reflected upon. Students were also given opportunities to address issues that they felt they did not quite understand. Discussion then took place to explore reasons for lack of understanding. This not only help students better understand materials but also provide feedback to me to improve future instructions. Summative assessments were done through lab reports, project design documents, project final reports, and exams. Data show that majority students' work met the learning objectives.

\section{Conclusions}

The solar energy curriculum has been established in the electrical engineering program at Grand Valley State University. Three learning modules were developed and successfully incorporated in the undergraduate curriculum. A course dedicated to photovoltaic systems were offered to graduate students and selected undergraduate students. The curriculum is supplemented by the SolaRescue program and Alternative Energy Club. Through both theoretical and practical learning, students gained the skills needed to succeed in the field of photovoltaics.

\section{References}

1. Ben Gallagher, (2017), “PV System Pricing H1 2017: Breakdowns and Forecasts”, Gtmresearch, Solar.

2. R. Margolis, D. Feldman, and D. Boff, (2017), “Q4 2016/Q1 2017 Solar Industry Update”, NREL/PR-6A20-68425.

3. IRENA (2017), “Renewable Energy and Jobs - Annual Review 2017”, International Renewable Energy Agency, Abu Dhabi.

4. Greg R. Smestad and Michael Gratzel, (1998), "Demonstrating Electron Transfer and Nanotechnology: A Natural Dye-Sensitized Nanocrystalline Energy Converter”, J. of Chemical Education, Vol. 75, pp 752-756.

5. Linear Technology, "LTC3106 - 300mA Low Voltage Buck-Boost Converter with PowerPath and 1.6 $\mu$ A Quiescent Current”, http://www.linear.com/product/LTC3106, (Accessed Jan. 2017).

6. L. Jiao, (2016) “Development of a Photovoltaic Emergency Power System”, American Society for Engineering Education Annual Conference and Exposition, New Orleans, LA, \#16826. 